\title{
Ion Dynamic Capture Experiments With The High Performance Antiproton Trap (HiPAT)
}

\author{
James Martin ${ }^{1}$, Raymond Lewis ${ }^{2}$, Suman Chakrabarti ${ }^{1}$, William H Sims ${ }^{1}$, \\ J Boise Pearson ${ }^{1}$ and Wallace E Fant ${ }^{3}$ \\ ${ }^{I}$ NASA MSFC, TD40, Huntsville, Alabama, 35812 \\ ${ }^{2} R$. Lewis Company (MSFC, TD40), Huntsville, Alabama, 35812 \\ ${ }^{3}$ Cortez III, Huntsville, Alabama, 35812 \\ (256) 544-6054, James.J.Martin@msfc.nasa.gov
}

\begin{abstract}
To take the first step towards using the energy produced from the matter-antimatter annihilation for propulsion applications, the NASA Marshall Space Flight Center (MSFC) Propulsion Research Center (PRC) has initiated a research activity examining the storage of low energy antiprotons. The High Performance Antiproton Trap (HiPAT) is an electromagnetic system (Penning-Malmberg design) consisting of a 4 Tesla superconductor, a high voltage electrode confinement system, and an ultra high vacuum test section. It has been designed with an ultimate goal of maintaining $10^{12}$ charged particles with a half-life of 18 days. Currently, this system is being evaluated experimentally using normal matter ions that are cheap to produce, relatively easy to handle, and provide a good indication of overall trap behavior (with the exception of assessing annihilation losses). The ions are produced via a positive hydrogen ion source and transported to HiPAT in a beam line equipped with electrostatic optics. The optics serve to both focus and gate the incoming ions, providing microsecond-timed beam pulses that are dynamically captured by cycling the HiPAT forward containment field like a "trap door". Initial dynamic capture experiments have been successfully performed with beam energy and currents set to $1.9 \mathrm{kV}$ and $23 \mu$ amps, respectively. At these settings up to $2 \times 10^{9}$ ions have been trapped during a single dynamic cycle.
\end{abstract}

\section{INTRODUCTION}

The annihilation of matter with antimatter represents the highest energy density of any known reaction, producing $10^{8} \mathrm{MJ} / \mathrm{g}$, or approximately 10 orders of magnitude more energy per unit mass than chemical based combustion. This potential makes it particularly attractive for those future deep space exploration missions that place high demands on propulsion systems's energy per unit mass. Over the years a number of potential antimatter utilization schemes have been suggested. These concepts range from lower performance high thrust/low specific impulse systems (comparable to nuclear thermal performance levels) to those that exhaust the annihilation products themselves (Morgan, 1982; Rider 1997). However, the more attractive concepts often referred to as "hybrids" - use antiprotons to initiate fusion reactions, potentially requiring fewer antiprotons since most of the energy is derived from the fusion burn (Kammash, 1992; Kramer, 2002)

The focus of this effort is to develop antiproton storage technology, a crucial capability for enabling many propulsion and commercial related applications. To accomplish this task, an experimental hardware program was established with the High Performance Antiproton Trap (HiPAT) (Martin, 2001; Martin, 2002) as the centerpiece. HiPAT system objectives include the following: 1) reaching storage quantity of $10^{12}$ antiprotons; 2) achieving particle lifetimes of 18 days; and 3) packaging the hardware in a portable design. To date, a majority of the hardware has been assembled and baseline system operation is being assessed through bench testing of normal matter ions. Normal matter is provided by two methods: electron guns (simple and easy to use); and external ion sources. The ion source is the method of choice, since the layout of this system more closely approximates the operations expected at an actual antiproton production facility with "packets" of antiprotons having pulse widths measured in 100's of nanoseconds. The ion sources can provide beam energies up to $20 \mathrm{kV}$ with a maximum current greater than $100 \mu \mathrm{mps}$ (for protons). For the present test sequence the source and electric fields are operated at $10 \%$ of their maximum. Dynamic trapping techniques have successfully demonstrated capture of approximately $10^{9}$ hydrogen ions; the data trending with simple models used to predict particle loading based on the timing sequence, trap electric/magnetic fields, and ion beam 
current. Modeling will be refined and trapping techniques expanded to examine multiple shot capture or "stacking" to increase the total number of ions stored within HiPAT.

\section{HARDWARE COMPONENTS}

The experimental setup consists of several primary components: the electromagnetic trap system (referred to as HiPAT), the beam line (a transport conduit for ions), and the ion source (which produces trappable monoenergetic ions). Figure 1 illustrates the basic layout of this system with key components identified.
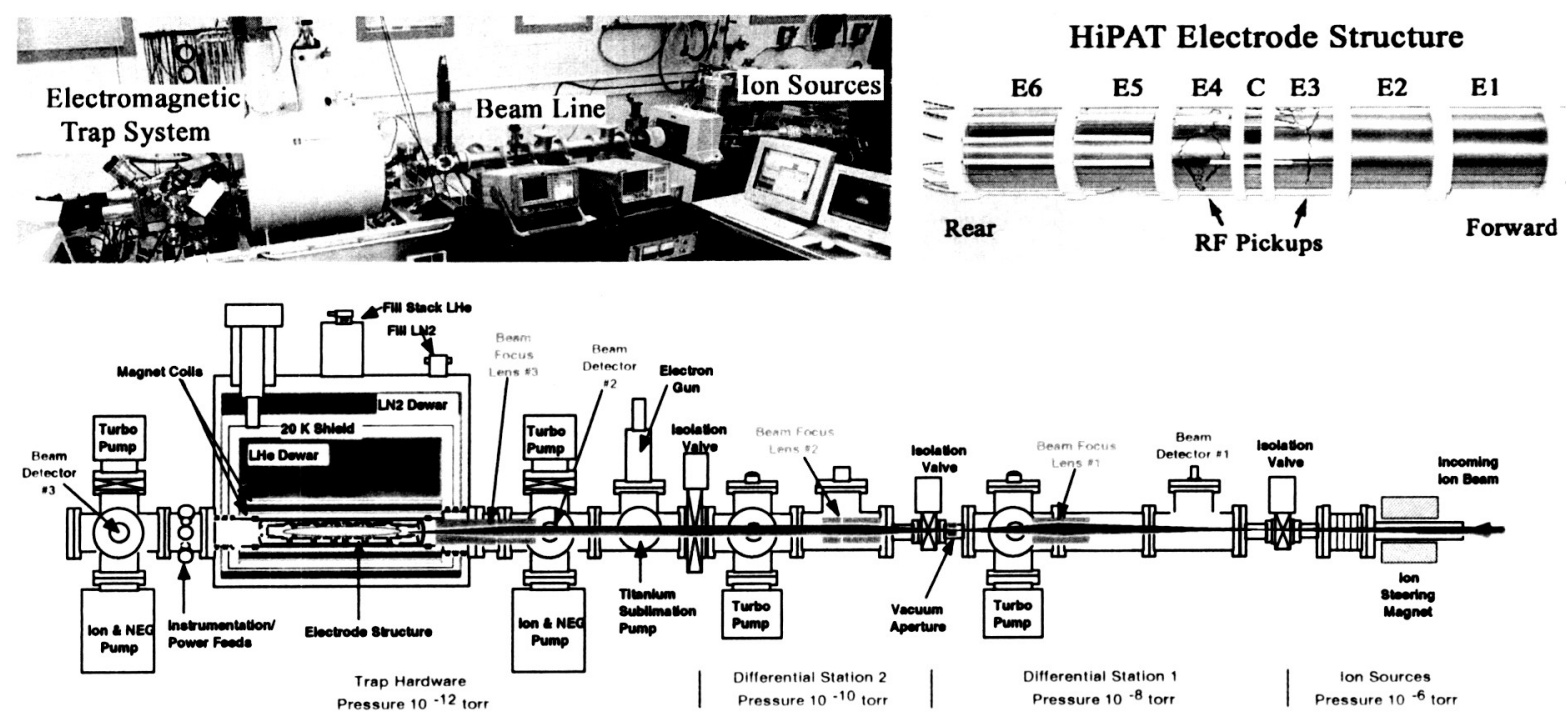

FIGURE 1. HiPAT Layout and Hardware Schematic.

\section{HiPAT Containment System}

The HiPAT has been designed as a portable ion containment device incorporating a number of subsystems: a superconducting magnet, ultra-high vacuum pumping equipment, high voltage electrode assembly, beam optics/sensors, and electronics to monitor and interact with trapped particles. The electronics/control systems are critical to the operation of the entire experiment and therefore are fed power from a large battery backup unit (providing up to 12 hours of autonomous operation). Liquid helium and nitrogen are supplied to the magnet system via portable dewars (e.g., helium replenishment is required approximately every 100 hours). The overall magnetic and electric fields were designed to contain up to 1 trillion charged particles resulting in design capabilities of 4 Tesla and 20 kilovolts, respectively. To successfully capture ions and hence load the trap, it was necessary to design a high-speed control circuit to rapidly change the voltage on the lead trap electrode. This was accomplished by using several high-speed transistor switches (BEHLKE HTS-301), which can ramp the voltage on electrode 1 between a "high" and "low" setting. The "high" setting, well above that of the incoming ion beam energy, can stop the ions from entering or leaving the trap once inside; while the "low" position is set just below the beam energy, allowing ions access to the interior of the trap.

This system can be transitioned very quickly, with a period approaching 0.1 microseconds. To minimize background neutral gas loads within HiPAT (to reduce radial diffusion loss of trapped ions), an ultra high vacuum system with turbo-, ion, non-evaporable getter, and titanium sublimation pumps is used. The combination of these pumps and the use of system conditioning have allowed vacuum levels as low as $5 \times 10^{-12}$ torr to be achieved (measured with Varian UHV-24p low x-ray limit ion gauges and a Spectra Microvision Plus residual gas analyzer). Significant effort went into conditioning the system to remove as much contamination from the system as possible. The typical sequence includes an initial high temperature bake out at an average temperature of $200^{\circ} \mathrm{C}$ (duration of 24 to 48 hours) followed by a 4 hour hydrogen glow discharge cleaning cycle (to remove more tightly bound materials), followed up by another 48 hours of $200+{ }^{\circ} \mathrm{C}$ bake out. The glow treatment (Dylla, 1988;Sasaki, 1991; Halama, 1990) makes use of hydrogen gas (metered into the system 
to maintain a pressure of roughly 10 mill-torr) and approximately 100 watts of radio frequency power applied to all electrodes and lenses: during this process the entire length of the beam line and trap glows brilliant purple. Based on the applied current, time and surface area of the components an ion dose rate greater than $10^{18}$ ions $/ \mathrm{cm}^{2}$ was applied.

\section{Beam Line and Detector Setup}

The beam line connecting the HiPAT to the ion source is constructed from off-the-shelf Conflat type vacuum hardware (with a nominal $10 \mathrm{~cm}$ tube diameter). This system is approximately 2 meters long, encompassing the vacuum pumping stations, electron gun, beam optics (Einzel focusing lenses), and isolation valves (to control the differential pumping when the ion beam is emitted). This system is also conditioned in the same fashion as the HiPAT, both with high temperature bake out and hydrogen glow discharge. During normal operation, vacuum levels range from $10^{-6}$ to $10^{-12}$ torr along its length. However, during beam injection the isolation valves open for approximately 2.5 seconds resulting in a momentary pressure rise within the trap (low $10^{-11}$ torr range) as neutral gas is propagated downstream from the ion source. A typical beam injection sequence is illustrated in figure 2 .

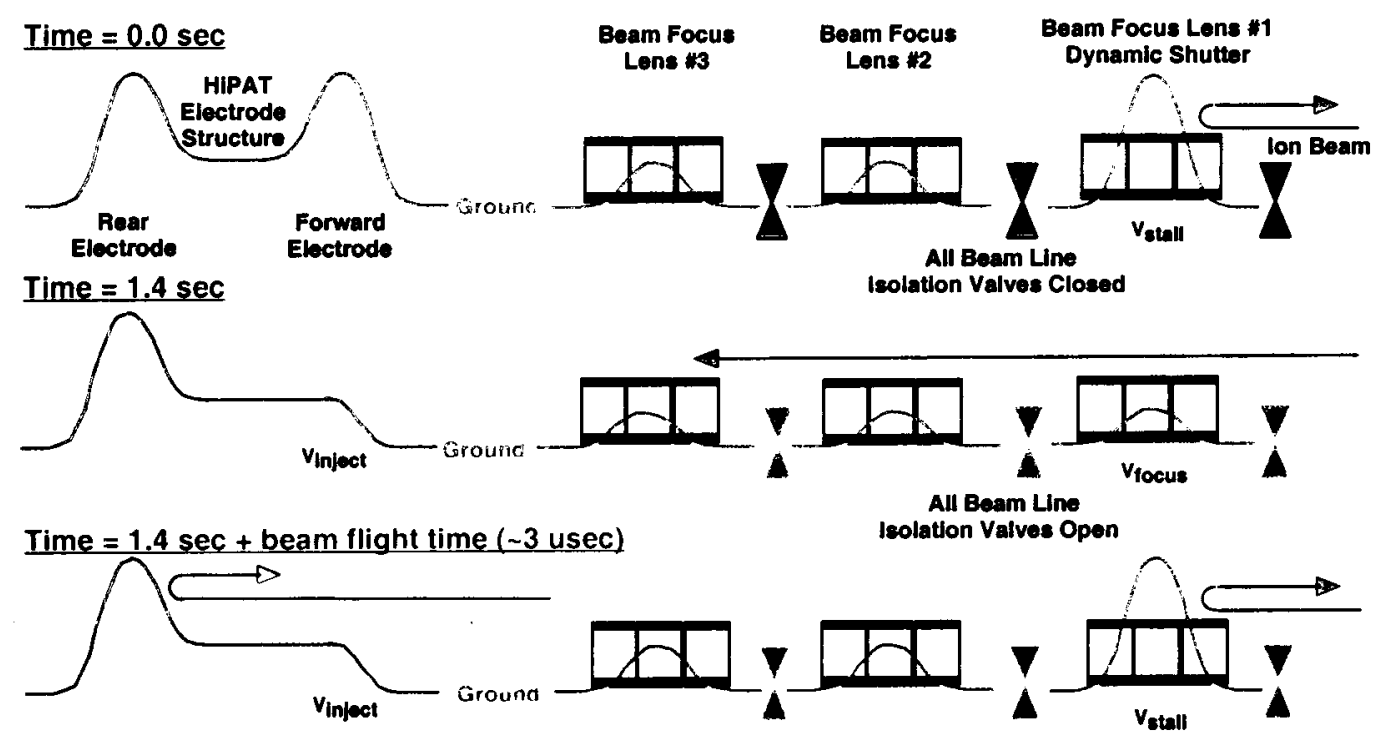

Time $=1.4 \mathrm{sec}+$ beam flight time + turnaround time $(-0.5 \mathrm{usec})$

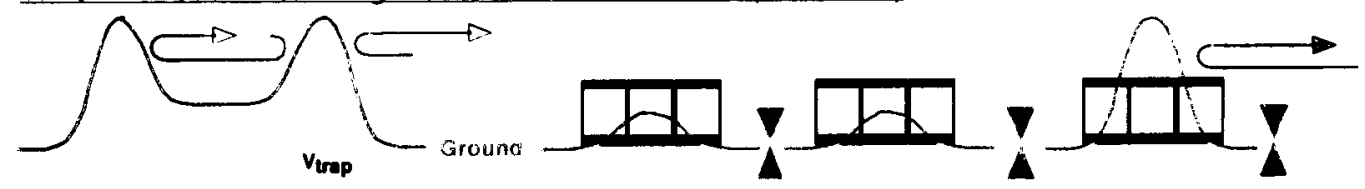

Time $=2.5 \mathrm{sec}$

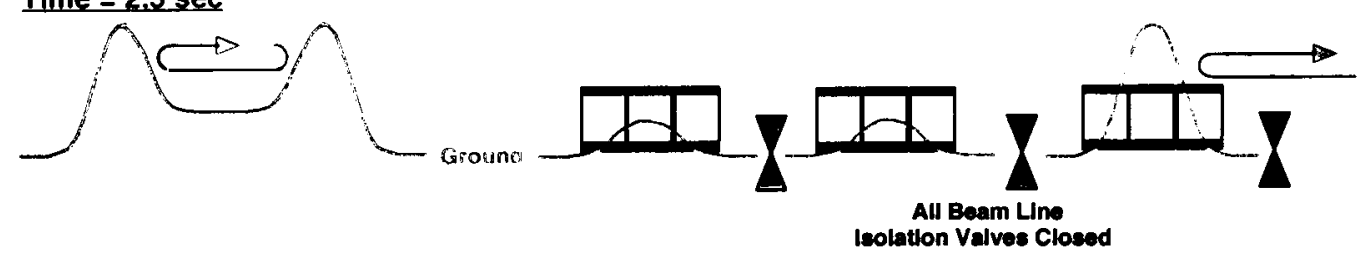

FIGURE 2. Typical Ion Capture Sequence.

To monitor the ion beam, several movable detectors are located along the beam line. When setting up the ion beam, the detectors are moved from an off-axis position onto the center of the beam line, with currents displayed on a Keithley model 485 Pico Ammeter. The first detector is positioned just downstream of the ion source, to observe the effect of adjustments made to the large ion source selection magnet (directs ions along 
the axis of the beam line). This detector consists of two dense wire meshes, which form a bull's-eye, providing sufficient information to determine the placement of the beam spot. The second detector is located just upstream of the HiPAT electrode structure (again a bull's-eye design) and provides spatial information regarding the beam center (focus) and intensity. The third detector is located on the downstream side of HiPAT and is identical in construction to the second detector. However, this last detector is located well out in the magnetic fringe field and therefore only $\sim 10 \%$ of the accepted beam can be measured due to beam divergence.

The primary need for these detectors is to monitor the setup and performance of a series of electrostatic lenses termed Einzel lens triplets. These lenses are strategically located to provide focusing of the ion beam, maximizing its transport along the beam line and acceptance into the trap magnetic fringe field (since matching the ion trajectory to the magnetic field lines minimizing reflection). The first Einzel lens is positioned downstream of the $1^{\text {st }}$ detector and serves two roles: 1) a short focal length lens that compresses the beam to a spot several millimeters in diameter allowing it to pass through a vacuum conductance aperture, and 2) a highspeed dynamic beam shutter. This shutter effect makes use of a high-speed transistor switch to bounce the voltage on the lens between a high or "stall" setting and a low or "focus" setting. This switching allows for a burst of ions with controlled pulse widths (often referred to as "beam spills") as short as 0.1 microseconds. The stall voltage is typically set to twice that of the beam energy and the focus at approximately half, meeting the requirements for a short focal length. The second lens is located approximately $60 \mathrm{~cm}$ downstream of the first one, with its voltage adjusted to maximize beam transmission to the detector just upstream of HiPAT. This lens's voltage is also set to approximately half of the beam energy producing a nearly parallel beam. The last lens is located just upstream of the trapping electrodes (in the strong fringe field of the superconductor) so that it can provide redirection to the parallel ion beam for matching the magnetic field lines. This last lens was found to optimize at approximately one third of the incident beam energy. During setup several small iterations on all lens voltage settings are made for producing maximum beam transport efficiency prior to running experiments. See Figures 1 and 2 for indications of placement and usage of these lenses.

\section{Ion Source System}

The ion source is a commercial unit produced by National Electrostatics Corporation (NEC): it consists of a positive ion source (Radio Frequency Oscillator) and a negative ion source (Source of Negative Ions by Cesium Sputtering (SNICS)). For the experiments described in this report, the positive RF system was the only source used. Typical settings used in the current study were a beam energy of approximately $2 \mathrm{kV}$ with beam currents up to $24 \mu \mathrm{amps}$ (at the trap entrance). The primary ion species used for experiments was $\mathrm{H}^{+}$(determined by the selector magnet); nevertheless, other hydrogen species $\left(\mathrm{H}_{2}{ }^{+}\right.$and $\left.\mathrm{H}_{3}{ }^{+}\right)$can also be generated and controlled. This ion source also required conditioning to achieve low vacuum levels: the bake out temperature is limited to $150^{\circ} \mathrm{C}$ due to restrictions on several mounted components and the ability to access all the "nooks and crannies". The source base vacuum was several time $10^{-9}$ torr, but was increased (by injecting hydrogen gas) to the mid-10 ${ }^{6}$ torr range during typical operation.

\section{TESTING DYNAMIC CAPTURE TECHNIQUES}

As a precursor to dynamic capture experiments, tests of the timing of the ion beam to the HiPAT electrode structure were performed. This information is used in setting up the timing and delay sequence for capture tests (to make sure the trap is sprung when ions are actually present). The timing was accomplished by using a Berkeley Nucleonics Corporation Model 555 Pulse/Delay Generator, which controlled the operation of all beam line components. This same timer was later integrated with the HiPAT forward electrode high-speed control system to enable dynamic captures.

\section{Hydrogen Beams Time of Flight Bench Test}

Several ion time-of-flight (TOF) and current measurements were made using the detectors both upstream and downstream of HiPAT. For these tests, the positive ion source was set to $1.9 \mathrm{kV}$ (as read on its power supply), producing a beam current of approximately 31 Hamps (at the first detector). The beam high-speed control system was set to deliver a $3 \mu \mathrm{sec}$ burst of ions. Figure 3 illustrates the typical diagnostic data recorded for one of these experiments. It shows both the voltage on the first Einzel lens (making a high-low-high stall/focus/stall cycle), and the second detector voltage signal as the ions strike it (the detector output is measured with an 
Agilent 54624A oscilloscope using a $1000 \Omega$ load as a shunt). This figure also includes data for beams of $\mathrm{H}_{2}{ }^{+}$ and $\mathrm{H}_{3}{ }^{+}$ions in addition to protons. By measuring the differences between the times-of-flight (TOF) of the different species, the square root of mass ratio can be verified. The following values are observed: $\mathrm{H}^{+}=2.92$ $\mu \mathrm{sec}, \mathrm{H}_{2}{ }^{+}=4.16 \mu \mathrm{sec}, \mathrm{H}_{3}{ }^{+}=5.0 \mu \mathrm{sec}$. Using the square root of the mass ratio (applicable since all species are at the same energy), the timing of $\mathrm{H}_{2}{ }^{+}$and $\mathrm{H}_{3}{ }^{+}$can be compared to that of $\mathrm{H}^{+}$, i.e. $\mathrm{H}_{2}{ }^{+}=\sqrt{2} * 2.92=4.13 \mu \mathrm{sec}$ and $\mathrm{H}_{3}{ }^{+}=\sqrt{3} * 2.92=5.06 \mu \mathrm{sec}$. The TOF values for the heavier hydrogen ions compare well to those measured. Also, the peak beam current measured (shown in figure 3 ) was $18 \mu \mathrm{amps}$ (calculated from $18 \mathrm{mV}$ signal $/ 1000$ $\Omega$ shunt load), which corresponds to a transmission efficiency of $\sim 70 \%$ along the beam line. This pulse derived beam current compares well with a steady-state reading of $20.1 \mu$ amps taken at the same location (second detector) using a Keithley Pico-ammeter Model 485. Note these reported beam intensity values have not been adjusted for either delta ray emission or screen porosity/area factors (the detectors were passive with no biasing to suppress delta rays). An additional set of tests was performed to evaluate the TOF between the second and third detectors for an $\mathrm{H}^{+}$beam. These data are illustrated in figure 4 and show that approximately $10 \%$ of the beam incident on the second detector is measured on the rear detector. However, this is expected since the beam will diverge significantly before it reaches the extreme rearward location of the third detector $(65 \mathrm{~cm}$ beyond the superconductor) without additional electrostatic focusing.

To correctly assess the beam energy from the recorded TOF data, allowances must be made for the variation in ion velocity along the beam line as it passes through each Einzel lens (climbing potential hills). To assess the variation along the axis of each electrostatic lens, a model was built using the TRAPEX code, which solves Laplace's equation to obtain the electric potential function. TRAPEX is a based on a SLAC electron gun design code called EGUN (Herrmannsfeldt, 1988). Accounting for the slowdown in ion velocity over the known path length between the second and third detectors results in a calculated beam energy of $1.97 \mathrm{kV}$, agreeing within $4 \%$ with the ion source power supply display.

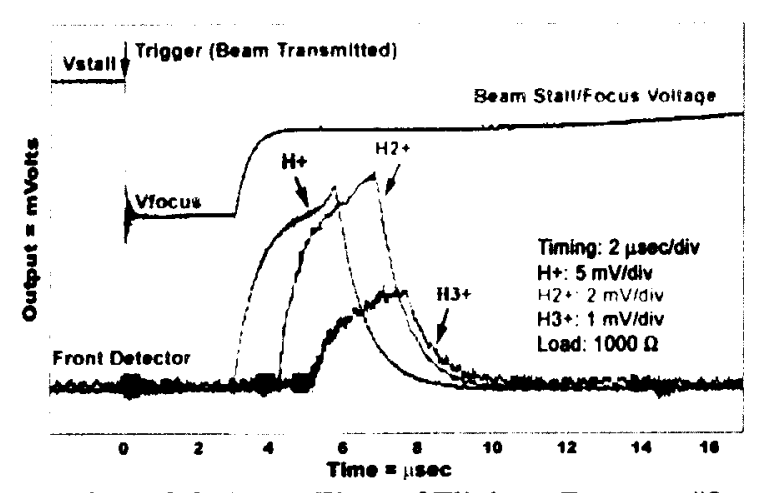

FIGURE 3. Beam Time of Flight to Detector \#2

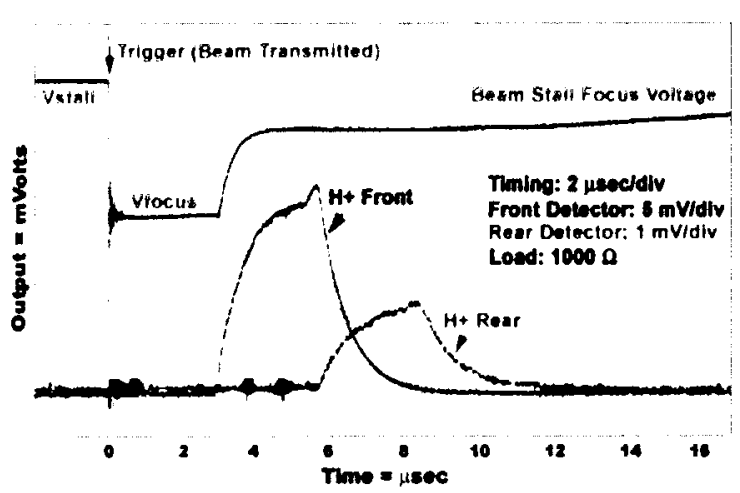

FIGURE 4. Beam Time of Flight to Detector \#3

\section{Beam Sequencing For Dynamic Capture}

With successful completion of beam gating experiments (precise spill times and pulse widths), the HiPAT forward electrode (E1) high-speed control circuitry was connected to the pulse/delay generator. To verify that dynamic capture does indeed occur, the timing delay for cycling trap electrode E1 was varied with respect to the incoming beam start time. Figure 5 illustrates these data, showing traces for the first Einzel lens (stall/focus voltage), the HiPAT El voltage and the second (HiPAT front) detector. The ion source was again operated with an $\mathrm{H}^{+}$beam energy of $1.9 \mathrm{kV}$, a current of approximately $26 \mu \mathrm{mps}$, and a beam spill width of $4 \mu \mathrm{sec}$. The trap electrode El has a cycle width of $1 \mu \mathrm{sec}$ with two voltage settings 1$)$ a high side ( $3 \mathrm{kV})$ to stop the beam and 2) a low side $(1.0 \mathrm{kV})$ to accept the beam. It is noted for these tests that the estimated residence time of an $\mathrm{H}^{+}$ion inside the trap is approximately $1.9 \mu \mathrm{sec}$ : therefore the $1 \mu \mathrm{sec}$ El cycle width would only capture approximately half the maximum number possible. Figure 6 shows electrode E1 delay varied over a range of $10 \mu \mathrm{sec}-$ an interval larger than the actual $\mathrm{H}^{+}$spill - to illustrate that dynamic capture is achieved when the beam spill and E1 cycle overlap. The number of trapped ions quickly plateaus at approximately $1.5 \times 10^{8}$ ions over a centrally located span of $2.0 \mu \mathrm{sec}$. A ramping in the number of trapped ions is seen in both the leading and trailing edges 
as a result of two experiment characteristics: 1) the finite cycling duration of $\mathrm{E} 1$, and 2) rounded edges due to instrumental resistor/capacitor time characteristics.

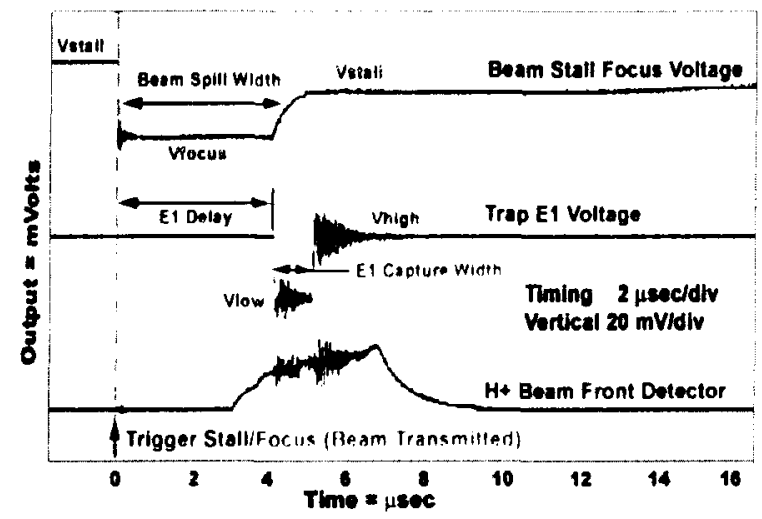

FIGURE 5. Event Sequencing.

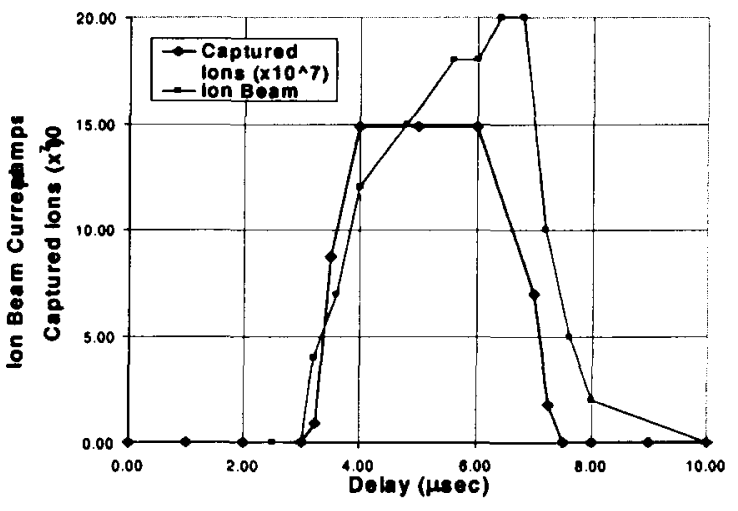

FIGURE 6. Dynamic Capture

These data also demonstrate that runaway secondary ionization within the negative wells (formed under the end electrodes) is essentially non-existent using the pulsed beam method. This ionization, due to the ions impinging on the positive potential region, is a major ion production method for techniques that use electron beams to fill the trap central well with ions. If significant ionization were to occur, it would be expected that for early E1 delays $(0$ to $3 \mu \mathrm{sec}$ ) ions would accumulate and be extracted from the trap. For long delays ( 7 to $10 \mu \mathrm{sec}$ ) no ions would be seen since the late cycling of $\mathrm{E} 1$ would allow any accumulated ions to escape prior to extraction. Nonetheless, test data indicated no early or late ions.

An estimate can be made for the number of electrons that would be produced by the pulsed beam. This number would be proportional to the product of the background gas density $(n)$, the interaction cross-section $(\sigma)$, the production path length (L), and the total number of ions (I); and can be expressed as:

$$
\mathrm{E}_{\text {total }}=\mathrm{n} \sigma \mathrm{I}_{\text {total }} \mathrm{L}_{\text {path }}
$$

Assuming typical test values of a number density of $2 \times 10^{6} \mathrm{~cm}^{-3}$, a cross-section of $1 \times 10^{-16} \mathrm{~cm}^{2}, 5 \times 10^{8}$ ions, and a path length of $10 \mathrm{~cm}$ (approximate path length of an end electrode), a single ionization would occur for a beam spill. This is hardly enough to cause the runaway reaction typical of the several second firing of an electron beam.

\section{Dynamic Capture Vs. Trap Well Depth}

A series of experiments was performed examining the number of ions dynamically captured as a function of the difference between the HiPAT central electric potential minimum (electrodes E2 to E5) and the incoming beam energy (this difference is called the well depth). As the well depth is decreased it should become easier to capture ions, since the ions decelerate as they climb the energy potential, lengthening their residence time in the trap. Operating conditions were similar to previous tests with the ion source set to $1.9 \mathrm{kV}$ and $24 \mu \mathrm{mps}$ at the trap entrance (adjusted for delta ray emission), electrodes E1 and E6 maintained at $3 \mathrm{kV}$, and the central electrodes (E2 to E5) ranged in unison to vary well depth. The beam spill was increased to $25 \mu \mathrm{sec}$ with a trap shutter of $20 \mu \mathrm{sec}$, providing up to $3 \times 10^{9}$ ions available for capture. The experimental data, illustrated in figure 7 , shows $1.8 \times 10^{8}$ ions captured at the deepest potential well, increasing to a peak of approximately $2.3 \times 10^{9}$ ions at a well depth $100 \mathrm{~V}$ lower than the nominal beam energy. An abrupt decrease in captured ions is observed beyond this maximum. Also included on this figure are the results of a simple model used to predict the number of trapped ions. The ion beam has a finite emittance $\left(\Delta \mathrm{x} \Delta \varepsilon_{\mathrm{x}} \sim 150 \pi \mathrm{mm}\right.$-mrad), which results in conversion of a portion of the axial velocity to cyclotron motion [Krauss-Vogt, 1988] as the beam enters the 4 Tesla field. The effect shows up as the correlation between radius and axial velocity shown in figure 8 , produced by a TRAPEX simulation. The model includes an r.m.s. radial beam profile also shown in figure 8 . 
The magnet fringe field converts typically $100 \mathrm{eV}$ of axial energy to cyclotron energy, resulting in a maximum yield when the central electric field is set to $100 \mathrm{eV}$ below the incident beam energy.

The model results agree with the trend of experimental data; however, it consistently under-predicts the experimental data except for the deepest potential well. Reasons for differences will continue to be investigated in future experiments, including these possibilities: ionization of background gas as hot ions cool; higher than expected beam line transmission and acceptance efficiency (fringe field); and peculiarities with the micro channel plate detector used to sample the extracted ion pulses.

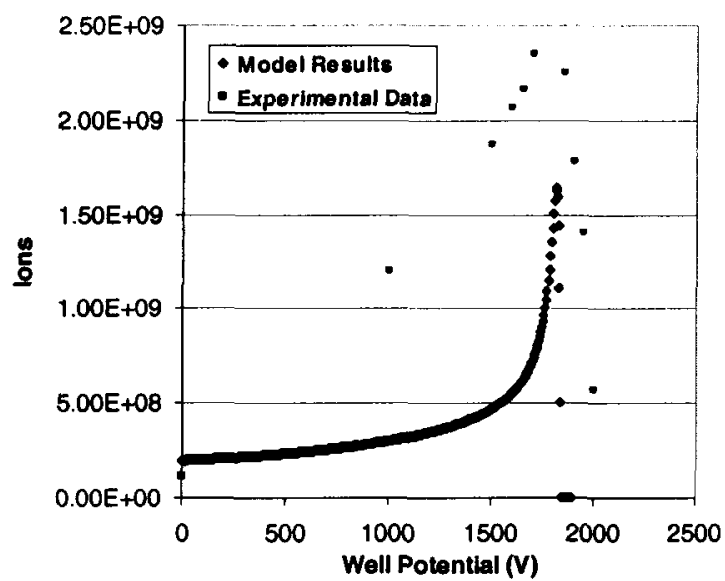

FIGURE 7. Captured Ions vs. Well Depth.

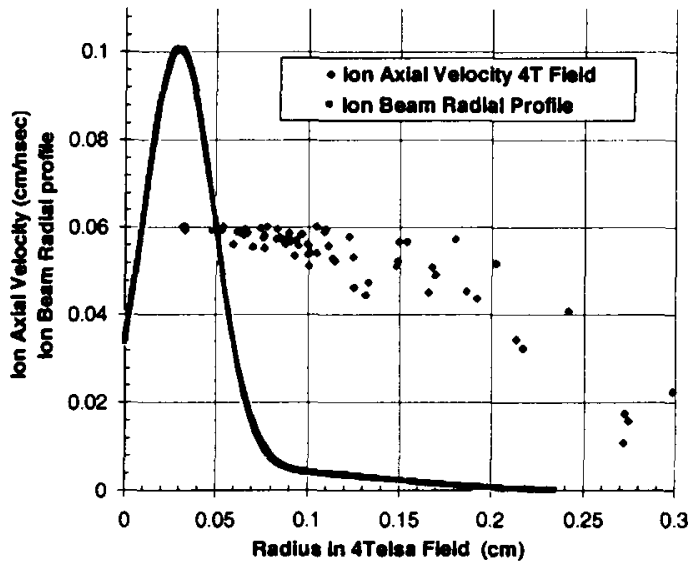

FIGURE 8. Variation of Beam Axial Velocity

\section{SUMMARY}

The HiPAT trap was configured and tested for dynamic capture of injected $\mathrm{H}^{+}$ions from an external source. Results confirm that that this objective was successfully accomplished, in that ions were captured only during the period where cycling of the trap electrodes coincided with the presence of the ion beam. Experiments were also performed examining the effects of trap well depth to determine settings for maximizing capture of incident ions. These tests show that as well depths were decreased the number of captured ions increased, achieving a maximum of $2.3 \times 10^{9}$ ions approximately $100 \mathrm{~V}$ below the beam energy. The simple model used for prediction of captured quantities showed the correct trend; however it consistently under-predicted the experimental data. Future work will involve 1) refining the dynamic capture modeling and instrumentation, 2) expanding the dynamic capture operation to include stacking techniques (multiple shots), and 3) increasing potentials up to 10 $\mathrm{kV}$ as an intermediate stop on a path to $20 \mathrm{kV}$.

\section{NOMENCLATURE}

$\begin{array}{lll}n & = & \text { background gas number density }\left(\mathrm{cm}^{-3}\right) \\ \sigma & = & \text { scattering cross-sections }\left(\mathrm{cm}^{2}\right) \\ \mathrm{L}_{\text {path }} & = & \text { production path length }(\mathrm{cm}) \\ \mathrm{E}_{\text {toal }} & = & \text { number of electrons produced } \\ \mathrm{I}_{\text {total }} & = & \text { number of primary ions }\end{array}$

\section{ACKNOWLEDGMENTS}

The authors wish to acknowledge the substantial contributions to the design, fabrication and assembly of hardware on this project made by Roger Harper.

\section{REFERENCES}


Dylla, H.F, "Glow Discharge Techniques for Conditioning High-Vacuum Systems," J. Vac Sci. Technology A, Vol 6, No. 3, pp 1276-1287, May/June 1988.

Halama, H.J., "Pretreatment and Conditioning of Storage Ring Vacuum Systems," AIP Conference Proceedings, Vol 200, Issue 1, pp 93-105, February 1990.

Herrmannsfeldt, W. B., "EGUN: an electron optics and gun design program," Tech. Rep. SLAC-0331, SLAC-331, SLAC-R-0331, SLAC-R-331, October 1988, 129pp., Stanford Linear Accelerator Center (SLAC), Stanford, CA, USA, Supersedes SLAC-0051, SLAC-0166, and SLAC-0226.

Kammash T., and Galbraith, D.L., "Antimatter-Driven Fusion Propulsion Scheme for Solar System Exploration," Journal of Propulsion and Power, Vol. 8, No. 3, May 1992.

Kramer, K.J., et. al, “Antimatter-Initiated Microfusion: Direct Energy Conversion for Propulsion Applications,” STAIF2002, edited by M.S. El-Genk, AIP Conf proceedings 608, AIP, New York, 2002, pp 787-788.

Krauss-Vogt, W., et al., "Emittance and Matching of ECR Sources," Nucl.. Inst. \& Meth. A268, 5, 1988.

Martin, J.J., et. al., "Design and Preliminary Testing of a High Performance Antiproton Trap (HiPAT)," STAIF-2001, edited by M.S. El-Genk, AIP Conf proceedings 552, AIP, New York, 2002, pp 931-938.

Martin, J.J., et. al., "Ion Storage Tests With The High Performance Antiproton Trap (HiPAT)," STAIF-2002, edited by M.S. El-Genk, AIP Conf proceedings 608, AIP, New York, 2002, pp 793-800.

Morgan, D.L., "Concepts for the Design of an Antimatter Annihilation Rocket," J. British Interplanetary Society, Vol 35.9, 1982.

Rider, T.H., "Fundamental Constraints on Large-Scale Antimatter Rocket Propulsion," Journal of Propulsion and Power, Vol. 13, Nov 3, 1997.

Sasaki, Y.T, "A Survey of Vacuum Material Cleaning Procedures: A subcommittee report of the American Vacuum Society Recommended Practices Committee," J. Vac Sci. Technology A, Vol 9, No. 3, pp 2025-2035, May/June 1991. 